\title{
Fractional Segmentation of White Matter
}

\author{
Simon K. Warfield ${ }^{1}$, Carl-Fredrik Westin ${ }^{1}$, Charles R. G. Guttmann ${ }^{1}$, \\ Marilyn Albert ${ }^{2}$, Ferenc A. Jolesz ${ }^{1}$, and Ron Kikinis ${ }^{1}$ \\ 1 Surgical Planning Laboratory, Department of Radiology \\ Harvard Medical School and Brigham and Women's Hospital \\ 75 Francis St., Boston, MA 02115 \\ FAX: $617-732-7963$ \\ \{warfield, westin, guttmann, jolesz, kikinis\}@bwh.harvard.edu \\ http://splweb. bwh.harvard.edu: 8000 \\ 2 Massachusetts General Hospital, Psychiatry/Gerontology (149-9124) \\ Bldg. 149, 13th Street, Charlestown, MA, 02129
}

\begin{abstract}
Abnormalities in the white matter of the brain are common to subjects with multiple sclerosis and Alzheimer's disease. They also develop in normal, asymptomatic, subjects and appear more frequently with age. Clinically, it is interesting to be able to differentiate between different disease states and to find markers which allow early diagnosis. Conventional spin echo (CSE) magnetic resonance imaging (MRI) is sensitive to these white matter changes and has frequently been applied to their study.
\end{abstract}

Previous approaches to investigate white matter abnormalities have often been reported to have difficulty distinguishing between normal gray matter and abnormal white matter due to their similar appearance in MRI. Earlier methods have also often generated binary classifications, reporting white matter as either normal or abnormal.

We have developed a new approach which first identifies the region of white matter using a template moderated spatially varying classification, and then estimates the degree of white matter abnormality present at each voxel of the white matter. This fractional segmentation allows us to preserve the heterogeneous characteristics of white matter abnormalities and to investigate both focal and diffuse white matter damage. We compute, from the fractional segmentation, a white matter spectrum showing the different levels of white matter damage present in each subject.

We applied this automated image segmentation method to over $996 \mathrm{MRI}$ scans of subjects affected by multiple sclerosis, 72 normal aging subjects and 29 scans of subjects with Alzheimer's disease. We investigated the ability to characterize these different subject groups based upon tissue volumes determined by spatially varying classification, and by the fractional segmentation of the white matter of each patient.

Keywords: automatic segmentation, brain, white matter, multiple sclerosis, Alzheimer's disease, normal aging. 


\section{Introduction}

Abnormal white matter of the brain is common to patients with one of several different diseases (including multiple sclerosis (MS) and Alzheimer's disease (AD)) and also appears in normal (asymptomatic) aging (NA) subjects. Better characterization of the nature of these white matter changes can help to improve our understanding of the biological processes at work. Clinically, it is interesting to be able to differentiate between different disease states and to find markers which allow early diagnosis. Conventional spin echo magnetic resonance imaging is sensitive to these white matter changes. MRI studies of patients and volunteers have indicated that the patterns of brain change associated with these processes are different. An important goal is to be able to quantitatively study these differences.

Many automated and semi-automated segmentation algorithms for quantitatively assessing these brain changes have been developed and validated. Most of these algorithms have aimed at determining a binary characterization of each voxel as one of a group of possible tissue classes. This approach has been limited by two factors. First, abnormal white matter is often isointense with normal grey matter and previous studies have been limited by the inability to discriminate between some abnormal white matter and normal grey matter [1,2]. Secondly, white matter damage appears as an heterogenous region of abnormal signal intensity but binarization of the segmentation treats all levels of signal intensity abnormality equally.

Segmentation methods for the assessment of white matter damage have in the past identified voxels in the region of white matter as either normal or as abnormal. This binarization of the state of white matter damage is at best a useful approximation to the actual underlying brain changes that cause the MRI signal intensity abnormalities.

Previous approaches for fractional segmentation of MRI have used geometric and probabilistic models. These methods have been developed in order to account for partial volume averaging to allow improved tissue volume estimates and to improve contrast between specific tissue types $[3,4,5,6]$.

Often these methods attempt to assign to each voxel a fractional volume of each tissue class modelled (usually based on the relative probability of the signal intensity occuring for each of the classes proposed to be present in a voxel). Our work differs from these methods in two ways. First, we use an anatomical localization procedure to identify the region of white matter and grey matter, and then look for a fractional segmentation in the region of white matter. Secondly, empirical estimation of a probability density function for the signal intensity distribution of abnormal white matter has the following characteristics. The most commonly occuring abnormality dominates the probability distribution regions of smaller and greater signal intensity are less probable. Consequently, a fractional segmentation based solely on the probability distribution of abnormal white matter signal intensity does not treat very bright regions differently from lower brightness regions (although they have different signal intensity characteristics, they can be equally probable). The use of relative weighting of the probability of white matter and abnormal white matter (a two class partial vol- 
ume assignment) can also be problematic because typical estimation methods for white matter probability give very low (or zero) estimates in the region of typically abnormal white matter. This then leads to binarization of the region of abnormal signal intensity, which we would like to avoid.

Mitchell et al. [7] described a method for constructing a white matter - gray matter spectrum by projecting CSE MRI signal intensity onto the first principle component of the white matter (WM) and gray matter (GM) cluster. Intensity normalization was based on the CSF cluster but the inability to assign meaning to specific locations along the line of projection restricted the method to the use of arbitrary units. This method used manually determined ROIs to investigate the WM-GM spectrum of individual lesions. Spectrums were shown for a few time points of a few lesions in a few patients, and highlighted the possibility of such approaches for characterizing abnormal white matter.

We have developed a new automated image segmentation algorithm which is more sensitive and specific for white matter damage and which allows for the investigation of different levels of signal intensity abnormality. Our approach uses automated white matter segmentation, and so can characterize the entire white matter region, not just easily identifiable lesions. Our method is able to generate a calibrated scale for the degree of white matter damage present at each voxel in the white matter region. The goal of this new segmentation method is to allow improved measures of white matter damage to be developed and to allow quantification of damage suspected to be present in so-called normal-appearing white matter.

Our approach to the assessment of fractional white matter damage can be characterized as a geometric feature space model. Unlike earlier geometric models which relied upon more than two tissue classes, our use of automated segmentation of the white matter allows us to assess white matter damage without regard to other tissue classes (such as CSF). This makes our overall approach of first tissue segmentation and then fractional segmentation more robust than previously reported approaches for white matter characterization.

In the following sections, the image segmentation algorithm is presented, and several approaches to characterizing white matter damage are proposed. The algorithm and different white matter characterizations were applied to $72 \mathrm{MRI}$ scans of normal aging subjects, 29 MRI scans of Alzheimer's disease subjects and 996 MRI scans involving 46 patients with multiple sclerosis.

\section{Materials and Method}

The image analysis is a two step process. First we generate a high sensitivity and specificity segmentation of the regions of white matter and grey matter. Then we generate a fractional segmentation of the region of white matter.

Nine hundred and ninety six MRI scans acquired during a previous study of the evolution of multiple sclerosis [8] were re-analyzed. Seventy two subjects participated in the study as part of a large study of normal aging [1]. There were 22 men and 50 women. All subjects provided informed consent consistent with the institutional IRB regulations. 
Twenty nine subjects were included in the study with dementia of the Alzheimer type. The diagnosis of probable AD was made in concordance with NINCDS/ADRDA criteria. All patients had a history of a gradually progressive decline in cognition, demonstrated by difficulty in social or occupational function and impairments in memory and at least one other area of mental ability.

The conventional spin echo magnetic resonance images used in this study were prospectively acquired on a GE Signa $1.5 \mathrm{~T}$ scanner. Each scan covered the entire brain with axial slices and each slice was acquired with an in-plane voxel size of $0.9375 \times 0.9375 \mathrm{~mm}^{2}$ and a slice thickness of $3.0 \mathrm{~mm}$.

\subsection{High Sensitivity and Specificity Tissue Class Segmentation}

Recent review papers $([2,9])$ have highlighted the importance of developing new automated methods for segmentation in the presence of the overlapping intensity distributions of abnormal white matter and normal tissues.

The segmentation method we use involves a sequence of operations. First is intensity based classification with intensity correction using the EM algorithm [10]. This normalizes the intensities so that different scans acquired at different times are directly comparable. The intracranial cavity (ICC) is identified with a semi-automatic method that has previously been described and validated [11]. The tissue classes identified inside the ICC which are segmented are CSF, white matter, gray matter and lesion. We then match a volumetric brain atlas to the subject with linear [12] and nonlinear registration [13], and resolve classification errors using anatomical context. We have previously described a general method for using anatomical context to resolve tissue class ambiguity due to overlapping intensity distributions [14]. The atlas is used to identify deep grey matter structures and to estimate the location of the cortical grey matter. It is then identified with region growing. The ability to segment cortical grey matter has previously been described and validated [15]. Our approach to the segmentation of deep gray matter structures has previously been described and validated [16].

We identify the white matter region by removing the gray matter structures and CSF tissue class from the ICC. The fractional segmentation described in the next section is then used to identify the level of white matter damage at every voxel in the white matter region.

\subsection{Fractional Segmentation of the White Matter Region}

The region of abnormal signal intensity associated with a focal lesion may contain areas of normal tissue as well as different histopathological components, such as edema, inflammation, gliosis, demyelination and axonal loss. Microscopic lesions of a size below the voxel resolution of the scanner occur in the normalappearing white matter, and constitue an 'invisible' lesion load which a binary segmentation is unable to detect, because of the relatively small effect upon signal intensity these have. The inability to account for these factors (changes in normal-appearing white matter, different pathological factors of lesions) has 
been highlighted as a limitation of existing techniques for measurement of lesion burden [2] and recognized as an important goal for increasing the accuracy and efficacy of quantitative analysis of MS lesions from MRI [9]. However, it is also recognized that if a lesion burden measure is unbiased, reproducible and related to disease activity it can be used as a reliable indicator of disease progression, even if the measure does not fully reflect the underlying pathology [9].

Our work described here aims to investigate the potential for characterizing the variation in white matter signal intensity observed in conventional spin echo images (PDW and T2W images). The model we use is based on a simple observation of the signal intensity characteristics of normal and abnormal white matter in CSE images. Lesions appear relatively bright in both PDW scans and T2W scans. Normal white matter appears darker than grey matter in PDW and T2W scans. Focal white matter abnormalities are often the brightest region of the white matter and diffuse white matter abnormalities often appear as regions of slightly less intense white matter signal intensity increase.

We propose a projection of the signal intensity variation of white matter in this two dimensional MR intensity space onto a line joining the signal intensity characteristics of the darkest white matter and the most bright white matter. We hypothesise that the darkest white matter is the "healthiest" white matter and the brightest white matter is the "most damaged" white matter and we use this linearization to define a mapping beween two dimensional MR intensity space and our measure of white matter damage (0 is most healthy, 1 is most damaged). We can then characterize the dual channel signal intensity properties of the white matter regions in terms of its level of "white matter damage".

The observed voxel values are modelled as being due to a combination of the fraction of each tissue type present in the brain over the region from which the voxel value is measured and a white noise process. Of course, this is a simplification. For instance, even healthy individuals have some intrinsic signal intensity variation in the white matter which is related to the structure of the white matter rather than to disease processes (for example, regions of tight white matter bundles, such as the corpus callosum, appear darker). Let $D$ be the voxel value that would be measured under ideal imaging conditions for a voxel containing maximum disease, and let $H$ be the value measured for healthy tissue. In the presence of noise and imaging artifacts, the observed dual echo signal intensity of a voxel will be $O^{\prime}$, randomly perturbed away from the ideal position. Let $O$ be the projection of an observed voxel $O^{\prime}$ onto the line joining $D$ and $H$ in the two channel feature space. We model the signal intensity at $O$ as being due to fractions of diseased, $f D$, and healthy, $(1-f) H$, tissues:

$$
O=f D+(1-f) H, f \in[0,1]
$$

This model includes the same information as a conventional binary classification (tissue classified as either healthy or diseased white matter) since a binary classification can be obtained from this model by thresholding at $\mathrm{f}=0.5$. Figure 1 is an illustration of the fractional segmentation of the white matter region of one image from a CSE MRI of the brain of one patient with multiple sclerosis. 


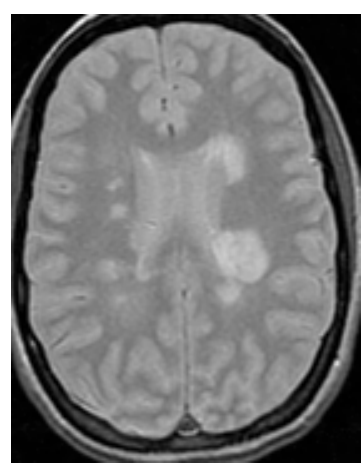

(a) Early Echo (PDw)

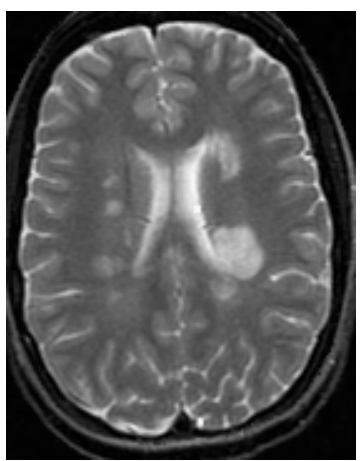

(b) Late Echo (T2w)

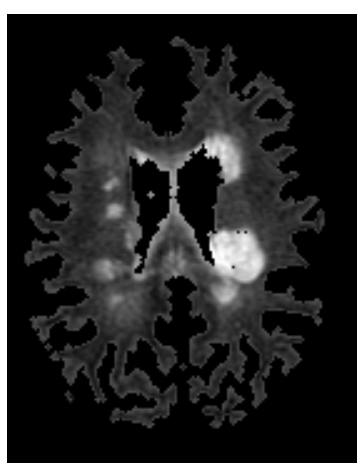

(c) Fractional Segmentation

Fig. 1. This figure illustrates the fractional segmentation of the white matter region from one slice of a subject with multiple sclerosis. Intensity in image (c) is proportional to the fraction of white matter damage estimated to be present at the voxel. Note the preservation of the lesion heterogeneity and the easy visualization of the regions of diffuse white matter damage.

\subsection{Analyzing the Distribution of White Matter Damage}

Once the white matter region has been identified and the fractional segmentation of the white matter region has been computed, we can study the distribution of the white matter damage as estimated by the fractional white matter segmentation, in order to gain a better understanding of the characteristics of white matter change.

One method for doing this is to compute a histogram of the fractional white matter segmentation. Normalizing this histogram generates a probability distribution function which gives the empirically determined probability that each level of white matter damage appears in the subject. We call this the white matter spectrum of the patient. It indicates the probability of different levels of white matter damage.

Computing a mean distribution (mean white matter spectrum) from the white matter fractional segmentation of a group of patients with AD gives an indication of the typical range of white matter abnormality present in patients with AD. Similarly, a mean white matter spectrum can be computed for normal subjects age matched to the AD subjects. Investigation of the differences between these distributions may indicate differences in the development of white matter abnormalities between these two groups. 


\section{Results}

We first present conventional volumetric tissue measurements derived by spatially varying classification of MRI of normal aging (NA) and Alzheimer's disease $(\mathrm{AD})$ subjects, and then show the white matter spectra of these and MS patients.

Figure 2 illustrates the differences between tissue volumes measured for normal aging and $\mathrm{AD}$ subjects. The $\mathrm{AD}$ subjects have lower relative white matter volume and higher relative lesion and CSF volumes than NA subjects and this effect is particularly pronounced with increasing age. The relationship of normal and abnormal white matter volume to ICC volume qualitatively reproduces that of an earlier study [1] which was carried out with a different image processing method, and so acts as a validation of the spatially varying classification technique used here.

Figure 3(a) shows the mean white matter spectrum computed from our databases of normal aging scans and AD scans, and the differences between these spectra in the region above the midpoint between healthy and diseased white matter $(\mathrm{f}=0.5)$. Figure $3(\mathrm{~b})$ shows the mean white matter spectrum of the normal aging subjects, the mean white matter spectrum derived from 996 scans of $46 \mathrm{MS}$ patients, and the difference between these spectra in the region above the midpoint between healthy and diseased white matter $(\mathrm{f}=0.5)$.

For comparison with the fractional segmentation distributions, Figure 4 shows normalized histograms of the $\mathrm{T} 2 \mathrm{w}$ intensity of the white matter and gray matter regions. The figure also shows the intensity distributions both before and after intensity normalization. Intensity normalization is achieved with the EM segmentation algorithm, which is a nonlinear, locally adaptive process. This figure shows that the intensity normalization process corrects for scanner and patient intensity variability, since the $\mathrm{T} 2 \mathrm{w}$ distributions after intensity correction are quite similar. Note that while intensity normalization is necessary in order to derive the fractional segmentation and to allow comparison of intensities from different subjects, it does not distinguish between artifactual intensity inhomogeneity and disease related intensity inhomogeneity, so that, for example, if the $\mathrm{T} 2 \mathrm{w}$ intensity of a disease process was uniformly brighter than a normal subject this difference would not be identified after intensity normalization. Although this does not appear to be a difficulty in practice, it is possible to consider intensity normalization schemes that attempt to separately identify artifactual intensity variations and intrinsic disease related intensity variations.

\section{Discussion}

The fractional segmentation of the white matter is a new descriptive mechanism for characterizing white matter change. It allows the visualization and quantification of both focal white matter lesions and diffuse white matter abnormality. Unlike binary classification, fractional segmentation preserves the inhomogeneity observed in the white matter. The fractional segmentation allows the construction of a white matter spectrum for each subject and for groups of subjects. 


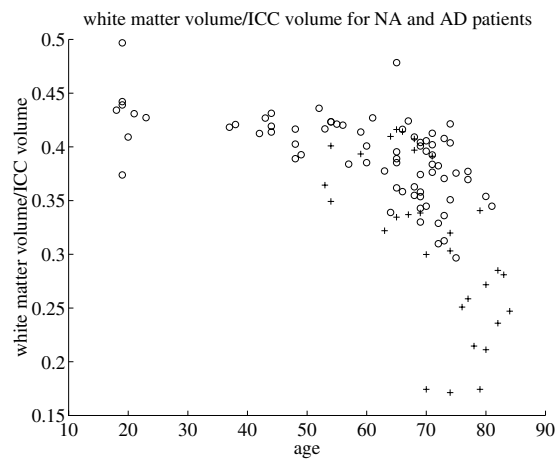

(a) Ratio of white matter volume to intracranial cavity (ICC) volume, as a function of age, for normal aging (NA, o) and Alzheimer's disease $(\mathrm{AD},+)$ subjects.

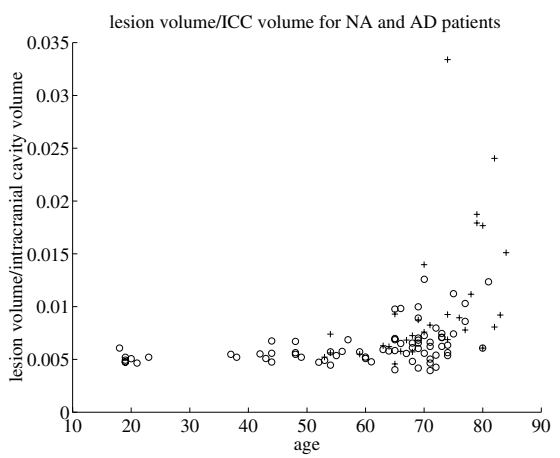

(b) Ratio of abnormal white matter volume to intrancranial cavity volume, as a function of age, for normal aging (NA, o) and Alzheimer's disease $(\mathrm{AD},+)$ subjects.

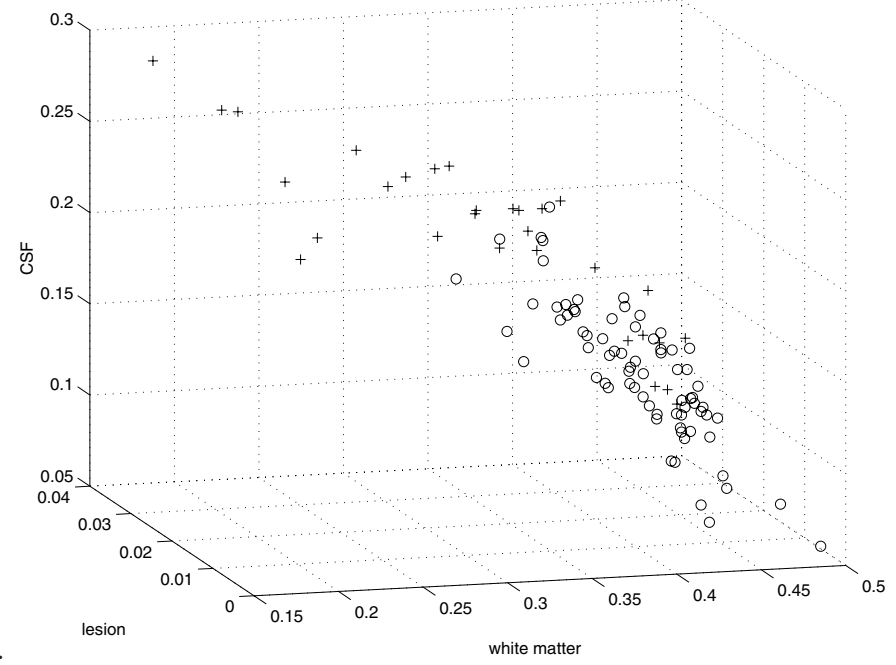

(c) Scatter plot of white matter, lesion and CSF tissue volume to ICC volume ratios for $\mathrm{AD}(+)$ and NA (o) subjects.

Fig. 2. Tissue volumes determined with spatially varying classification for normal aging and Alzheimer's disease subjects. The relationship of normal and abnormal white matter volume to ICC volume as a function of age qualitatively reproduces that of an earlier study carried out with a different image processing technique, and so acts as a validation of the spatially varying classification technique. It appears to be possible to distinguish many of the $\mathrm{AD}$ subjects from the NA subjects on the basis of the CSF/ICC, abnormal white matter/ICC and normal white matter/ICC ratios. 

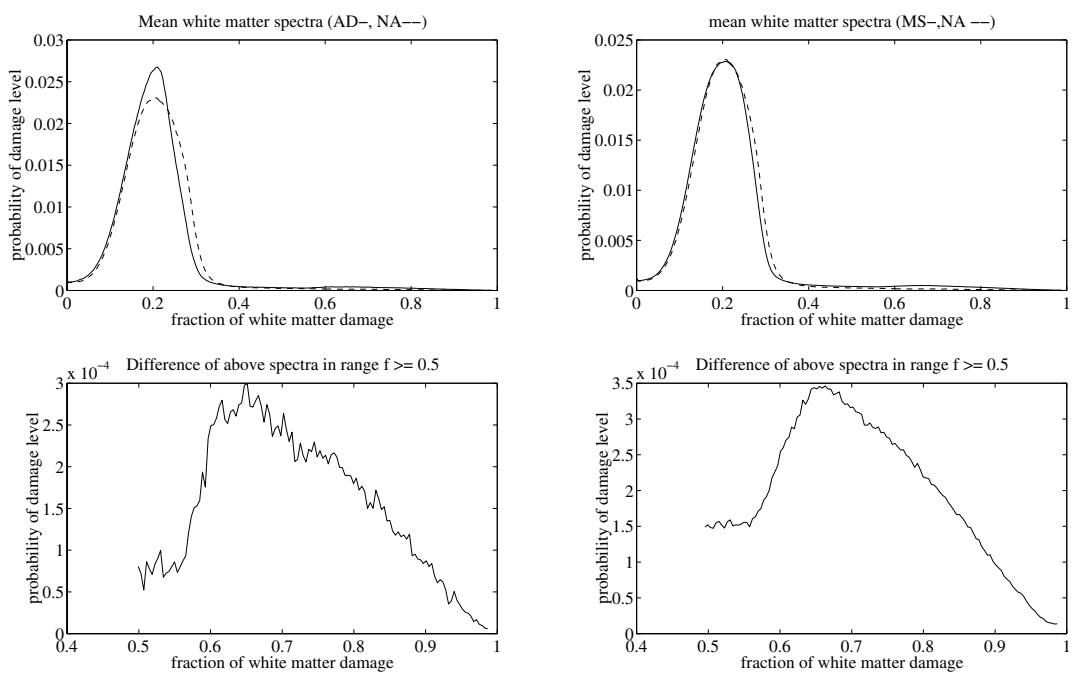

(a) Comparison of $\mathrm{AD}, \mathrm{NA}$ mean white matter spectra.

(b) Comparison of MS, NA mean white matter spectra.

Fig. 3. Fractional segmentation of the white matter region from normal aging, Alzheimer's disease volunteers and multiple sclerosis patients was carried out. The mean white matter spectrum was calculated by averaging the normalized fractional segmentation histogram of each subject. The difference of the mean spectra is shown in detail for the damage level above 0.5 . The nonzero differences raise the possibility that the white matter spectrum is indicative of different disease states.

Further analysis is necessary to determine if different diseases give rise to characteristic white matter spectra.

Tissue volumes determined by template moderated spatially varying classification indicate that a loss of normal white matter and an increase in CSF and abnormal white matter is characteristic of Alzheimer's disease. The relationship between age, CSF volume, and normal and abnormal white matter volume determined with this method (Figure 2) qualitatively reproduces earlier results [1]. This indicates that localization of the white matter region with spatially varying classification is a reasonable initial step prior to the computation of the fractional segmentation of this region.

We are currently investigating methods for differentiating between normal aging and Alzheimer's disease, and for identifying different sub-types of multiple sclerosis based on an analysis of the white matter spectra. 

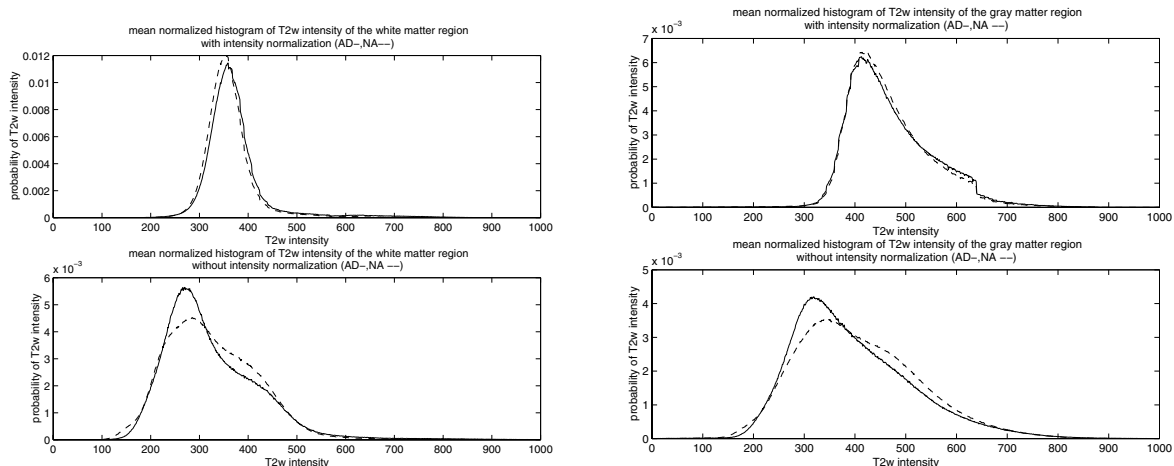

Fig. 4. This figure illustrates white matter and gray matter T2w MRI intensity distributions from the regions of white matter and gray matter of normal aging and Alzheimer's disease volunteers. This figure allows comparison of the T2w intensity distribution both before and after intensity normalization.

\section{Acknowledgements}

This investigation was supported (in part) by a grant from the National Multiple Sclerosis Society (SW) and by NIH grants P41 RR13218-01, R01 RR11747-01A, P01 CA67165-03 and P01 AG04953-14.

\section{References}

1. C. R. G. Guttmann, F. A. Jolesz, Ron Kikinis, R. J. Killiany, M. B. Moss, T. Sandor, and M. S. Albert, "White matter changes with normal aging", Neurology, vol. 50, pp. 972-978, 1998. 63, 64, 68, 70

2. Robert I. Grossman and Joesph C. McGowan, "Perspectives on Multiple Sclerosis", American Journal of Neuroradiology, vol. 19, pp. 1251-1265, August 1998. 63, 65, 66

3. Hwan Soo Choi, David R. Haynor, and Yongmin Kim, "Partial Volume Tissue Classification of Multichannel Magnetic Resonance Images - A Mixel Model", IEEE Transactions On Medical Imaging, vol. 10, no. 3, pp. 395-407, 1991. 63

4. David C. Bonar, Kirt A. Schaper, Jon R. Anderson, David A. Rottenberg, and Stephen C. Strother, "Graphical Analysis of MR Feature Space for Measurement of CSF, Gray-Matter, and White-Matter Volumes", Journal of Computer Assisted Tomography, vol. 17, no. 3, pp. 461-470, 1993. 63

5. Yi-Hsuan Kao, James A. Sorenson, Mark M. Bahn, and Stefan S. Winkler, "DualEcho MRI Segmentation Using Vector Decomposition and Probability Techniques: A Two-Tissue Model", Magnetic Resonance in Medicine, vol. 32, no. 3, pp. 342357, September 1994. 63

6. H. Donald Gage, Peter Santago, and Wesley E. Snyder, "Quantification of Brain Tissue Through Incorporation of Partial Volume Effects", in SPIE Medical Imaging VI: Image Processing, 1992, vol. 1652, pp. 84-96. 63 
7. J. Ross Mitchell, Craig Jones, Stephen J. Karlik, Karen Kennedy, Donald H. Lee, Brian Rutt, and Aaron Fenster, "MR Multispectral Analysis of Multiple Sclerosis Lesions", JMRI, vol. 7, pp. 499-511, May/June 1997. 64

8. Charles R. G. Guttmann, Sungkee S. Ahn, Liangge Hsu, Ron Kikinis, and Ferenc A. Jolesz, "The Evolution of Multiple Sclerosis Lesions on Serial MR.", AJNR, vol. 16, pp. 1481-1491, 1995. 64

9. M. Filippi, M. A. Horsfield, P. S. Tofts, F. Barkhof, A. J. Thompson, and D. H. Miller, "Quantitative assessment of MRI lesion load in monitoring the evolution of multiple sclerosis", Brain, vol. 118, pp. 1601-1612, 1995. 65, 66

10. W. M. Wells, R Kikinis, W. E. L. Grimson, and F. Jolesz, "Adaptive segmentation of MRI data", IEEE Transactions On Medical Imaging, vol. 15, pp. 429-442, 1996. 65

11. Ron Kikinis, Martha E Shenton, Guido Gerig, John Martin, Mark Anderson, David Metcalf, Charles R G Guttmann, Robert W McCarley, William E Lorenson, Harvey Cline, and Ferenc Jolesz, "Routine Quantitative Analysis of Brain and Cerebrospinal Fluid Spaces with MR Imaging", Journal of Magnetic Resonance Imaging, vol. 2, pp. 619-629, 1992. 65

12. Simon K. Warfield, Ferenc Jolesz, and Ron Kikinis, "A High Performance Computing Approach to the Registration of Medical Imaging Data", Parallel Computing, vol. 24, no. 9-10, pp. 1345-1368, September 1998. 65

13. Simon K. Warfield, Andre Robatino, Joachim Dengler, Ferenc A. Jolesz, and Ron Kikinis, Nonlinear Registration and Template Driven Segmentation, chapter 4, pp. 67-84, Progressive Publishing Alternatives, 1998. 65

14. Simon K. Warfield, Michael Kaus, Ferenc A. Jolesz, and Ron Kikinis, "Adaptive Template Moderated Spatially Varying Statistical Classification", in MICCAI 98: First International Conference on Medical Image Computing and ComputerAssisted Intervention. October 11-13 1998, pp. 231-238, Springer Verlag. 65

15. Simon Warfield, Joachim Dengler, Joachim Zaers, Charles R.G. Guttmann, William M. Wells III, Gil J. Ettinger, John Hiller, and Ron Kikinis, "Automatic identification of Grey Matter Structures from MRI to Improve the Segmentation of White Matter Lesions", Journal of Image Guided Surgery, vol. 1, no. 6, pp. 326-338, 1995. 65

16. Dan V. Iosifescu, Martha E. Shenton, Simon K. Warfield, Ron Kikinis, Joachim Dengler, Ferenc A. Jolesz, and Robert W. McCarley, "An Automated Registration Algorithm for Measuring MRI Subcortical Brain Structures", NeuroImage, vol. 6, pp. $12-25,1997.65$ 